\title{
Association of particular HLA class II alleles, haplotypes and genotypes with susceptibility to IDDM in the Belgian population
}

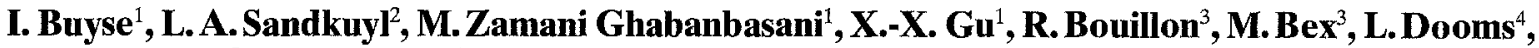 \\ M.-P. Emonds ${ }^{5}$, M. Duhamel ${ }^{6}$, P. Marynen ${ }^{1}$, J.-J. Cassiman ${ }^{1}$ \\ ${ }^{1}$ Center for Human Genetics, University of Leuven, Belgium \\ ${ }^{2}$ Voorstraat 27, Delft, The Netherlands \\ ${ }^{3}$ Endocrinology Department, University of Leuven, Belgium \\ ${ }^{4}$ Pediatrics Department, University of Leuven, Belgium \\ ${ }^{5}$ Blood Transfusion Center, Leuven, Belgium \\ ${ }^{6}$ Innogenetics, N. V., Gent, Belgium
}

Summary Using a highly discriminatory DNA typing technique, based on the polymerase chain reaction and reverse dot blot hybridization, more refined results were obtained on the association of particular HLA class II alleles, haplotypes and genotypes with insulin-dependent diabetes mellitus in the Belgian population. The previously reported predisposing effect for the DRB $1 * 0301$ encoded DR3 serologic specificity was confirmed and could be assigned to the DRB3 $* 0200$ encoded DR52b serologic specificity. A second high risk haplotype, DRB1*0401-DQB1*0302 encoding the DR4-DQ8 serologic specificity, accounted for increased susceptibility both in the total insulin-dependent diabetic population and among DR4-positive patients. Moreover, we found that these
DR4 associated DRB1 and DQB1 alleles act as independent risk factors. A possible role for the DPB1 locus can be rejected since the observed predisposing effect for DPB $1 * 0202$ probably occurred due to linkage disequilibrium of this allele with DRB1*0301. Particular extended haplotypes accounted for the decreased relative risk observed for the DR2, DR11 and DR13 serologic specificities. The highest relative risk was observed for those DQA1/DQB1 genotypes, allowing for the formation of $4 \mathrm{SS}$ $\left(\mathrm{DQ} \alpha^{\mathrm{Arg} 52+} / \mathrm{DQ} \beta^{\mathrm{Asp} 57-}\right)$ heterodimers. [Diabetologia (1994) 37: 808-817]

Key words Insulin-dependent diabetes mellitus, HLA, class II, PCR, SSO typing.
IDDM results from the autoimmune destruction of the pancreatic insulin-producing beta cells. Concordance rates for monozygotic twins ( $36 \%$ ), HLA-identical and -haplo-identical siblings ( $13 \%$ and $4.5 \%$, respectively) suggest a multifactorial predisposition for this disease, the genetic factor being only partly determined by the HLA genes [1,2].

Received: 26 November 1993

and in revised form: 21 March 1994

Corresponding author: Professor J.-J. Cassiman, Center for Human Genetics, University of Leuven, Campus Gasthuisberg, $0 \& N 6$, Herestraat 49, B-3000 Leuven, Belgium

Abbreviations: aa, Amino acid; Asp, aspartic acid; Arg, arginine; SSO, sequence-specific oligonucleotide; $P C R$, polymerase chain reaction; NBT, nitroblue tetrazolium chloride; BCIP, 5-Bromo4-chloro-3-indolyl phosphate; RR, relative risk; $d f$, degrees of freedom; HO, null hypothesis; IDDM, insulin-dependent diabetes mellitus
HLA genes are encoded in the human MHC complex on chromosome $6 \mathrm{p} 21.3$. Strong linkage disequilibrium exists within this complex of genes but rare recombination events during evolution gave rise to different HLA haplotypes in different ethnic groups [3-5]. Among Caucasians, HLA class II DR 3 and DR4 alleles are positively associated with IDDM, while DR2 alleles have been shown to confer protection to the development of the disease [2]. A stronger association with homozygosity for particular DQB1 alleles encoding an aa other than Asp at position 57 was originally found by Todd et al. [6] and confirmed in other studies $[7,8]$. Since then, interest has focused on the DQ subregion and a stronger association was found when both DQB1 and DQA1 loci were examined [9]. Characteristically, DQA1 alleles conferring susceptibility encode Arg at aa position 52. Khalil et al. [10] demonstrated that rather the combined presence of $\mathrm{DQ} \beta^{\mathrm{Asp57-}}$ (S) and DQ $\alpha^{\text {Arg52+ }}$ (S) alleles confer the highest risk of developing IDDM. A model was pro- 
posed suggesting a dose effect for the number of susceptible DQ $\alpha \beta$ (SS) heterodimers, encoded in cis or trans [11].

In this study 211 IDDM patients and 205 healthy control subjects, all Caucasians from Belgian origin, were typed for the DRB1, DRB3, DRB4, DRB5, DQA1, DQB1 and DPB1 alleles, using a previously described rapid and non-isotopic SSO typing assay, based on the PCR and reverse dot blot hybridization [12]. We evaluated a possible association of the class II alleles and their extended haplotypes and genotypes with IDDM.

\section{Materials and methods}

Patients and control subjects. Blood from a total of 211 unrelated IDDM patients, all Caucasians of Belgian origin was collected at the paediatric and adult endocrinology units of the University Hospital of Leuven. All children and adolescents had typical clinical IDDM, while in adults IDDM was confirmed by very low C-peptide levels after glucagon administration (maximum $<0.1 \mathrm{mmol} / \mathrm{l}$ ) or by the presence of anti-islet auto-antibodies. Eighty-nine patients were female and 122 were male; the mean age at disease onset was 16.5 years (range $1-53$ years). Our control population consisted of 205 healthy blood donors, all Caucasians of Belgian origin. They were collected from the Blood Transfusion Center of Leuven and had no personal or family history of IDDM.

$P C R$ amplification and SSO typing. All patients and control subjects were typed for the DQA1, DQB1, DPB1, DRB1, DRB3, DRB4 and DRB5 alleles as described previously [12]. Briefly, the polymorphic second exons of the DQA, DQB, DPB, DRB genes were amplified by the PCR and subsequently hybridized to a total of 11 DQA1, 18DQB1,23 DPB1 and 31 DRB SSOs using a rapid and non-isotopic reverse dot blot hybridization assay. A DR4DRB1 group-specific amplification andhybridization was also included to increase the resolution for particular DRB1*04 homoor heterozygous persons. The originally described DRB and DPB1 hybridization protocols were adapted to yield a reverse hybridization assay on membrane-based strips with SSOs immobilized in a line-wise fashion and the initially described chemiluminescence detection protocol was adapted to yield a colorimetric visualization of positive hybridization signals, using the NBT/BCIP substrates (Innogenetics N. V., Gent, Belgium).

For the DQA1 gene, at the time we started this study the DQA $1 * 0302$ and DQA $1 * 0104$ alleles had not yet been described and therefore the DQA1*0101/0104 and DQA1*0301/0302 alleles could not be distinguished and were grouped in this study as DQA $1 * 0101$ and DQA $1 * 0301$, respectively. For the DQB 1 gene, the recently described $\mathrm{DQB} 1 * 0606$ allele [13] could not be distinguished from DQB1*0605. The recently defined DQB1*0304 [14], DPB1*2201 [15], DPB1*2301 [16], DRB1*0303 [17], DRB1*1409 and DRB1*1410 $[16,18]$ alleles were each unambiguously defined by a unique combination of SSOs and could therefore be included in this study. For the DRB locus, the DRB1*1501/1503 and DRB1*1601/1602 alleles were not distinguished by our combination of SSOs and were grouped as DRB1*1501 and DRB1*1601, respectively. Because typing of the four expressed DRB genes is done simultaneously, combinations of alleles differing only at aa position 86 could not be distinguished. Therefore, the alleles DRB $1 * 1501$ / 1502, DRB1*0302/0303, DRB1*1101/1104, DRB1*1301/1302, DRB1*1401/1407, DRB1*1402/1406, DRB1*0801/0805,
DRB3*0201/0202 were grouped as DRB1*1500, DRB1*0302, DRB1*1101, DRB1*1301, DRB1*1401, DRB1*1402, DRB $1 * 0801$ and DRB3*0200, respectively.

A DRB1 locus-specific PCR, amplifying all DRB1 alleles except the DR2, DR7 and DR9 groups, was achieved using the previously described DRB1 primer CRX37 (5'GAATTCCCGCGCCGCGCT-3') [19], matching a unique DRB1 sequence in the intron 3' of exon 2, in combination with the conserved DRB-1 primer (5'-GATCCTTCGTGTCCCCACAGCACG-3') used in our assay for the co-amplification of the four DRB genes [12]. Genomic DNA $(0.5 \mu \mathrm{g})$ or $2.5 \mu \mathrm{l}$ of a lysed blood sample was amplified in a total volume of $50 \mu \mathrm{l}$ containing $50 \mathrm{mmol} / 1 \mathrm{KCl}, 10 \mathrm{mmol} / 1$ Tris- $\mathrm{HCl}(\mathrm{pH} 8.3$ ), $0.01 \%$ gelatin, $1.5 \mathrm{mmol} / / \mathrm{Mg}^{2+}, 200 \mu \mathrm{mol} / \mathrm{l}$ of each dATP, dCTP, dGTP (Pharmacia LKB Biotechnology, Uppsala, Sweden), $180 \mu \mathrm{mol} / 1$ of dTTP (Pharmacia LKB Biotechnology, Uppsala, Sweden), $20 \mu \mathrm{mol} / \mathrm{l}$ of biotin-11-dUTP (Sigma Chemical Company, St. Louis, Mo., USA), $0.2 \mu \mathrm{mol} / 1$ of each primer and 0.5 unit of Thermus aquaticus DNA polymerase (Perkin-Elmer Cetus Corp., Norwalk, Conn., USA). The amplification was carried out on a DNA Thermal Cycler (Perkin-Elmer Cetus Corp). An initial denaturation step of 5 min at $95^{\circ} \mathrm{C}$ was followed by 32 cycles of incubation for $1 \mathrm{~min}$ at $95^{\circ} \mathrm{C}, 1 \mathrm{~min}$ at $55^{\circ} \mathrm{C}, 1 \mathrm{~min}$ at $72^{\circ} \mathrm{C}$. A final extension step of $10 \mathrm{~min}$ at $72^{\circ} \mathrm{C}$ was added. In our study this DRB1 typing was used to discriminate the DRB $1 * 0802 / \mathrm{DRB} 1 * 0804 \mathrm{al}-$ leles and the DRB1*0301/DRB1*0302/DRB1*0303 alleles for heterozygotes carrying the DRB $3 * 0301$ allele. A DR2-DRB1 group-specific amplification was developed using the DR2DRB1 primer (5'-TTCCTGTGGCAGCCTAAGAGG-3') of the 11 th International Histocompatibility Workshop, matching the unique DRB1 sequence that characterizes the DR2 alleles, in combination with the conserved DRB-2 primer (5'GCCGCTGCACTGTGAAGCTCTC-3') used in our assay for the coamplification of the four DRB genes [12]. The amplification mixture and PCR conditions were identical to the DRB1 locusspecific amplification except for Taq polymerase, used in a concentration of 1 unit $/ 50 \mu$ amplification mixture. This DR 2 groupspecific analysis was performed in our study to analyse the DRB1 alleles in DR2 homozygotes and particular DR2 heterozygotes.

Statistical analysis. RR is an odds ratio: (number of patients with allele/number of patients without allele)/(number of control subjects with allele/number of control subjects without allele) [20]. $P$-values were calculated by the Fisher Exact Test [21] and corrected for the number of tests carried out by the use of the Bonferoni correction $[22,23]$. RRs were calculated for different alleles, haplotypes and genotypes. In the analysis of the alleles and haplotypes, we assume that individual alleles and haplotypes at a given locus constitute independent risk factors. RRs and $p$-values were only calculated for those alleles or haplotypes that were counted at least 10 times in the total (control + IDDM) population.

In each group of control subjects and IDDM patients, DQA1-DQB1-DRB1 haplotypes were deduced from subjects who were homozygous for at least two of three loci. The obtained results fitted with those expected from the reported linkage disequilibria [4, 24], except for two previously undescribed haplotypes. DRB1-DRB3 haplotypes were deduced from DRB1 (DRB3 associated) or DRB3 homozygotes and from DRB3/non-DRB3 heterozygotes. An analogous strategy was followed to deduce the DRB1-DRB5 haplotypes. No DRB1DRB3 or DRB1-DRB5 haplotypes that did not follow the established linkage disequilibrium were found [4].

Differences in RRs within groups of alleles were calculated via a likelihood ratio test. In this approach, two likelihoods are calculated on the observed data: the first likelihood is calculated under the assumption that all alleles of a given group convey the 


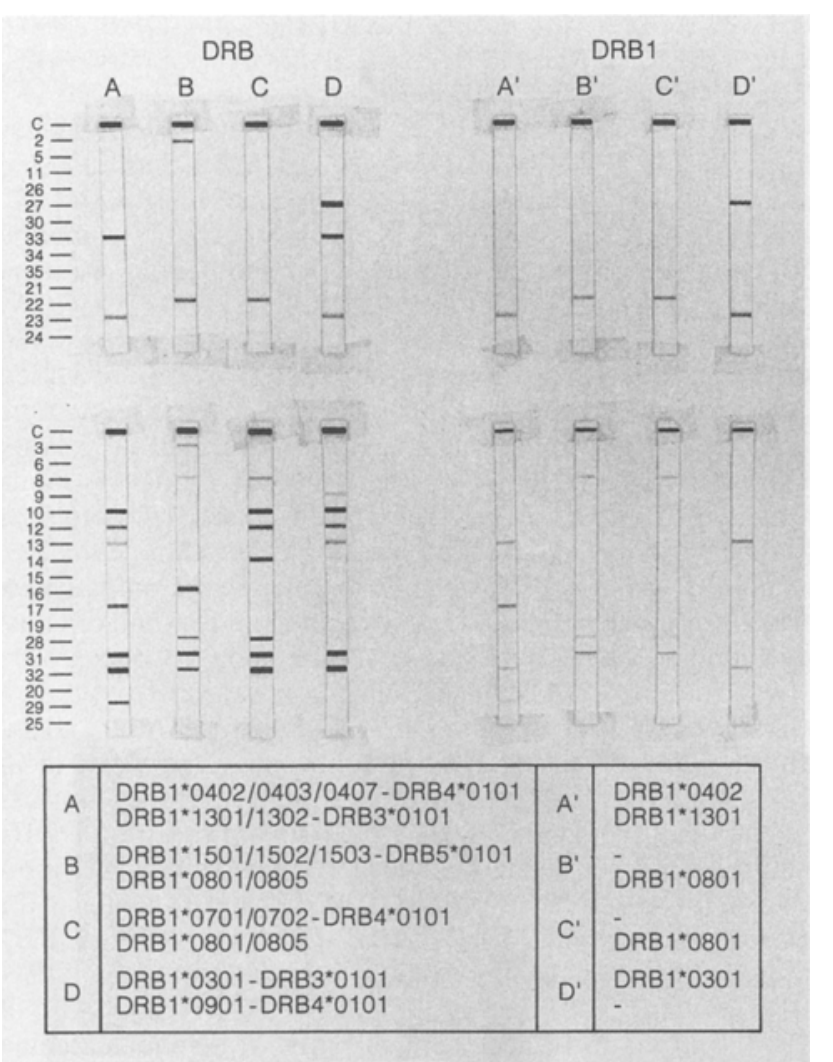

Fig.1. Examples of DRB1 locus-specific typing results. DNA of four heterozygous subjects was amplified for the DRB $(\mathrm{A}, \mathrm{B}, \mathrm{C}$, D) or DRB1 (A', B', C', D') genes, using the DRB-1/DRB-2 (12) or DRB-1/CRX37 primer pairs, respectively. PCR products were subsequently hybridized to a number of membrane-bound locusspecific SSOs. Positive hybridization signals were visualized using a colorimetric (NBT/BCIP based) detection reaction

same RR, while the second likelihood is calculated under the assumption that each allele within that group confers a specific $\mathrm{RR}$, which may be different from the RR of the other alleles. By taking twice the difference of the natural logarithms of these likelihoods, one obtains a statistic that will approximately follow a chi-square distribution, with the appropriate number of $d f$.

When a high- or low-risk allele was identified, we subsequently analysed the possibility of subdividing that risk allele more accurately by comparing the risks for two-locus haplotypes containing that particular allele. The statistical evaluation involved a chi-square test with the appropriate number of $d f$. While this method allows the definition of high- and low-risk haplotypes, it does not distinguish between the effects due to linkage disequilibrium between the loci and the effects due to independently acting risk alleles that occur together in a haplotype. To make such distinctions, we applied a stepwise likelihood-ratio test. The following scenarios were evaluated: $i)$ neither allele in the haplotype conveys any risk ( $\mathrm{HO})$, ii) only an allele at one of the loci conveys risk, there is no disequilibrium between the loci (one extra $d f$ over HO), iii) both loci convey risk, but they are not in linkage disequilibrium (the alleles constitute independent risk factors) (two extra $d f$ over $\mathrm{HO}$ ), iv) an allele at one of the loci conveys risk, the other allele is not a separate risk factor, but is in linkage disequilibrium with the first allele (two extra $d f$ over HO) and v) both loci convey risk, and they are in linkage disequilibrium with each other (four extra $d f$ over $\mathrm{HO}$ ).

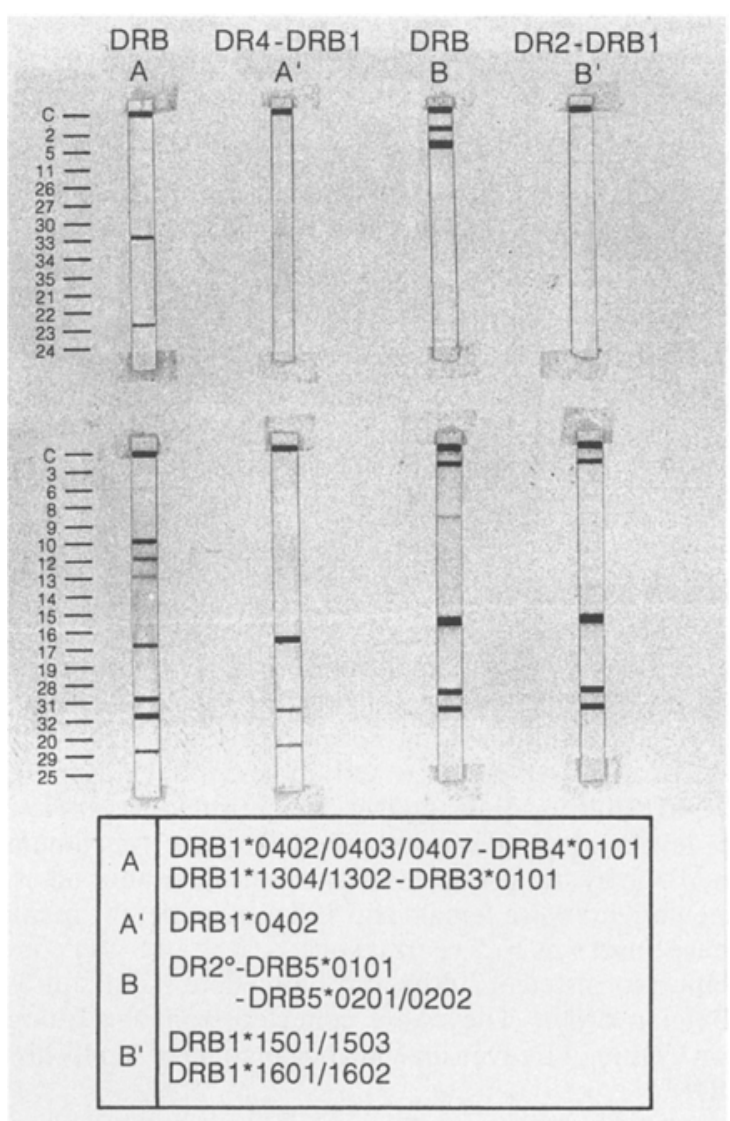

Fig.2. Examples of DR4-DRB1 and DR2-DRB1 group-specific typing results. DNA of two heterozygous subjects was amplified for the DRB (A, B) and DR4-DRB1 (A') or DR2-DRB1 (B') genes, using the DRB-1/DRB-2 and DR4-1/DRB-2 or DR2DRB1/DRB-2 primer pairs, respectively. PCR products were subsequently hybridized to a number of membrane-bound locus-specific SSOs. Positive hybridization signals were visualized using a colorimetric (NBT/BCIP based) detection reaction. DR $2^{\circ}$ : for DR2 homozygote subjects, the presence or allitic identity of the DRB1 genes cannot be detected using the DRB typing protocol

\section{Results}

DRB1 locus-specific and DR2-DRB1 group-specific analysis. In our initial assay [12], all four expressed DRB genes were amplified and typed together. This allows for a very rapid and simple analysis but complete subtyping becomes impossible for particular heterozygotes, encoding DRB1 alleles that differ only at aa position 86 . Also, no discrimination could be made between the DRB $1 * 03$ alleles for persons carrying the DRB3*0301. allele. To allow for further analysis of these allelic combinations, we developed a DRB1-specific PCR. This additional analysis was applied to discriminate the DRB1*0802/DRB1*0804 alleles and the DRB1*03 alleles in the presence of the DRB $3 * 0301$ allele. The existence of recombined DR2 DRB1-DRB5 haplotypes or haplotypes lacking the DRB1 or the DRB5 gene has been described previously [25-27]. A DR2-DRB1 
Table 1. Distribution of DQA1 alleles among IDDM patients and healthy control subjects

\begin{tabular}{|c|c|c|c|c|c|c|c|}
\hline \multirow[b]{2}{*}{ DQA1* } & \multirow[b]{2}{*}{$\operatorname{Arg}^{52}$} & \multicolumn{2}{|c|}{$\operatorname{IDDM}(n=211, \mathrm{a}=420)$} & \multicolumn{2}{|c|}{ Control $(n=205, \mathrm{a}=409)$} & \multirow[b]{2}{*}{ RR } & \multirow[b]{2}{*}{$p$} \\
\hline & & $n$ & $\%$ & $n$ & $\%$ & & \\
\hline$\overline{0101}$ & - & 43 & 10.2 & 62 & 15.2 & 0.638 & N.S. \\
\hline 0103 & - & 6 & 1.4 & 38 & 9.3 & 0.141 & $1.1 \times 10^{-6}$ \\
\hline 0201 & - & 19 & 4.5 & 55 & 13.4 & 0.305 & $2.92 \times 10^{-5}$ \\
\hline 0301 & + & 164 & 39.0 & 50 & 12.2 & 4.600 & $<10^{-15}$ \\
\hline 0601 & + & 0 & 0 & 1 & 0.24 & N.T. & N.T. \\
\hline $\operatorname{Arg}^{52+}$ & & 325 & 77.4 & 167 & 40.8 & 4.957 & $<10^{-15}$ \\
\hline
\end{tabular}

The alleles DQA1*0401/0601 could not be distinguished in one control subject and two IDDM patients, encoding DQA1*0201 on the second chromosome. For these subjects, only the DQA $1 * 0201$ allele was included in the statistical analysis. Therefore, a total of 420 diabetic and 409 normal chromosomes were compared. All DQA1 alleles that were not counted at least 10 times in the total (control + IDDM) population were not tested. $p$-values were corrected for the number of DQA1 alleles tested. $n$ : Number of IDDM patients and control subjects; a: Number of diabetic and control chromosomes. N.T.: not tested, N.S.: not significant

Table 2. Distribution of DQB1 alleles among IDDM patients and healthy control subjects

\begin{tabular}{|c|c|c|c|c|c|c|c|}
\hline \multirow[b]{2}{*}{ DQB1* } & \multirow[b]{2}{*}{$\mathrm{Asp}^{57}$} & \multicolumn{2}{|c|}{$\operatorname{IDDM}(n=207, \mathrm{a}=414)$} & \multicolumn{2}{|c|}{ Control $(n=205, \mathrm{a}=410)$} & \multirow[b]{2}{*}{ RR } & \multirow[b]{2}{*}{$p$} \\
\hline & & $n$ & $\%$ & $n$ & $\%$ & & \\
\hline 0501 & - & 40 & 9.7 & 54 & 13.2 & 0.705 & N.S. \\
\hline 0502 & - & 5 & 1.2 & 11 & 2.7 & 0.443 & N.S. \\
\hline 0503 & + & 2 & 0.5 & 8 & 2 & 0.244 & N.S. \\
\hline 0504 & - & 0 & 0 & 0 & 0 & & \\
\hline 0601 & + & 0 & 0 & 5 & 1.2 & & \\
\hline 0602 & + & 2 & 0.5 & 49 & 12 & 0.036 & $<10^{-11}$ \\
\hline 0603 & + & 6 & 1.4 & 33 & 8 & 0.168 & $4.35 \times 10^{-5}$ \\
\hline 0604 & - & 17 & 4.1 & 20 & 4.9 & 0.835 & N.S. \\
\hline 0605 & - & 2 & 0.5 & 7 & 1.7 & & \\
\hline 0201 & - & 156 & 37.7 & 82 & 20 & 2.419 & $1.52 \times 10^{-7}$ \\
\hline 0301 & + & 23 & 5.6 & 81 & 19.8 & 0.239 & $3.6 \times 10^{-9}$ \\
\hline 0302 & - & 139 & 33.6 & 34 & 8.3 & 5.590 & $<10^{-11}$ \\
\hline 0303 & + & 7 & 1.7 & 21 & 5.1 & 0.319 & N.S. \\
\hline 0304 & - & 0 & 0 & 0 & 0 & & \\
\hline 0401 & + & 0 & 0 & 0 & 0 & & \\
\hline 0402 & + & 15 & 3.6 & 5 & 1.2 & 3.045 & N.S. \\
\hline $\mathrm{Asp}^{57-}$ & & 359 & 86.7 & 208 & 50.7 & 6.339 & $<10^{-11}$ \\
\hline
\end{tabular}

$p$-values were corrected for the number of DQB1 alleles tested. All DQB1 alleles that were not counted at least 10 times in the total (control + IDDM) population were not tested. Four IDDM patients were not typed for DQB1. Therefore, a total of

group-specific amplification and hybridization was performed for DR2 heterozygotes carrying an allele that is identified by SSODRB16 and for DR2 homozygotes. Results of DRB1 and DR2-DRB1 group-specific typing results are shown in Figures 1 and 2.

Distribution of $D Q A 1$ alleles. As described previously [10], alleles encoding $\mathrm{Arg}^{52+} \mathrm{DQ} \alpha$ chains are susceptibility alleles (Table 1). A statistical test was carried out to evaluate whether there is a significant heterogeneity among the RRs of the various alleles encoding $\mathrm{Arg}^{52}$ positivity. The result yielded a chi-square value of 21.04 $(2 d f)$, which implies that homogeneity of risks within the $\mathrm{Arg}^{52+}$ group could be rejected with a $p$-value of
207 IDDM patients was included in the statistical analysis. $n$ : Number of IDDM patients and control subjects, a: Number of diabetic and control chromosomes. N.S., not significant

0.00003. Among IDDM patients, the frequencies of the DQA1*0301 $\left(\mathrm{Arg}^{52+}\right)$ and the DQA1*0102, DQA1*0103, DQA1*0201 ( $\mathrm{Arg}^{52-}$ ) alleles were significantly increased and decreased, respectively.

Distribution of $D Q B 1$ alleles. The distribution of the 16 DQB1 alleles defined in this study is shown in Table 2. As described previously [6-8], alleles encoding Asp ${ }^{57-}$ $\mathrm{DQ} \beta$ chains are susceptibility alleles (Table 2 ). Homogeneity among the RRs of the various alleles within the $\mathrm{Asp}^{57-}$ group could be rejected with a chi-square of $52.20(4 d f)$, corresponding to a $p$-value less than $10^{-6}$. Among IDDM patients, the frequencies of the DQB1*0201, DQB1*0302 (Asp $\left.{ }^{57-}\right)$ and the DQB1* 
0301, DQB1*0602, DQB1*0603 $\left(\mathrm{Asp}^{57+}\right)$ alleles were significantly increased and decreased, respectively.

Distribution of DPB1 alleles. Unresolved typing results were obtained for a total of four control subjects who were not included in the statistical analysis. The same was true for a previously undefined DPB $1 * 4601$ allele that we detected in one control subject [28]. The DPB $1 * 0401$ was the most frequent allele in the two populations. A significant increase $(\mathrm{RR}=16.66$, $p=0.0012$ ) of the rare DPB $1 * 0202$ allele in the IDDM patient population was found. In general, DPB1 alleles are not in linkage disequilibrium with $D R B$ or DQA/DQB alleles. However, the increased risk conferred by DPB $1 * 0202$ seems to be secondary to linkage disequilibrium with the increased DRB $1 * 0301$ allele in the IDDM population. Indeed all 17 IDDM patients carrying a DPB $1 * 0202$ allele were DRB $1 * 0301$ positive, although this does not mean that the two alleles were encoded on the same chromosome. However, 11 of 17 were DRB $1 * 0301$ homozygous and therefore at least $64.7 \%$ (in contrast to the $11.9 \%$ expected from the allele frequency of DRB $1 * 0301$ ) of the DPB $1 * 0202$ alleles were associated with the DRB1*0301 allele, clearly indicating a non-random association between these two alleles. The one DPB $1 * 0202$ positive control subject was DRB1*0301 negative.

Distribution of DRB alleles. The distribution of the 33 DRB1 and 3 DRB5 alleles defined in this study is shown in Table 3. Unresolved typing results (DRB1*0405/0408 = DRB1*0405/0405, DRB $1 * 0401 /$ $0405=$ DRB1*0408/0409, DRB1*1201/DRB1*0101DRB $1 * 0102$ ) were obtained for three IDDM patients and one control subject and were not included in the statistical analysis. The DRB1*1101/DRB1*1102 and DRB1*1301/DRB1*1305 alleles were pooled as DRB1*1100 and DRB1*1300, respectively and these groups of alleles were compared as such between the two populations. Because a similar protective effect was observed for the individual alleles that were pooled in the two groups and because the frequencies of DRB1*1102 and DRB1*1305 were low (data not shown), it is unlikely that this had any influence on the outcome of the analysis. Because no significant effects were observed for the different DR4 alleles, except for DRB1*0401, and because the frequencies of the individual alleles were rather low (data not shown), we decided to pool all DRB1*04 subtypes, except for DRB1*0401 (defined as DRB1*0400) and to analyse them separately from DRB $1 * 0401$.

Among IDDM patients, the frequency of the DRB1*0301 and DRB1*0401 alleles was significantly increased. A significant decrease of the DRB $1 * 1500$, DRB1*0701, DRB1*1100 (mainly caused by DRB1*1101, data not shown), DRB1*1300 (mainly caused by DRB1*1301, data not shown) alleles was also seen.
Comparison of DRB3 alleles revealed no significant association with IDDM. Thus, we analysed whether particular high or low risk DRB1 associations could be further defined by considering the DRB1-DRB3 haplotypes. Therefore, the DRB1-DRB3 haplotypes for those DRB1 alleles that were in linkage disequilibrium with more than one DRB3 allele (DRB1*0301 and DRB1*1300) were compared (Table 4). Subdivision of the DRB1*0301 haplotype into a DRB3*0101 and a DRB3*0200 group showed an increase of the DRB1*0301-DRB3*0200 haplotype in the IDDM group. It should be mentioned that RRs differ from those presented in Table 3. Here, the RRs were calculated by comparison with the non-DRB $1 * 0301$ group. Homogeneity among the RRs for the different DRB1*0301-DRB3 haplotypes within the DR3 group could be rejected with a chi-square of $19.30(1 d f)$, corresponding to a $p$-value of 0.000011 . Thus, although a susceptibility role for DRB $1 * 0301$ was found in the Caucasian population by several groups $[2,29,30]$, this effect could be further defined by analysing DRB1DRB3 extended haplotypes for this allele. Analogously, we analysed if the protective effect of DRB $1 * 1300$ could be further defined by considering the DRB1DRB3 extended haplotypes. For the DRB1*1300 alleles, the DRB $3 * 0101$ associated haplotype was significantly decreased in the IDDM patient population (data not shown). Again, RRs differ from those presented in Table 3, as they were calculated by comparison with the non-DRB $1 * 1300$ group. Homogeneity among the RR for the different DRB $1 * 1300-D R B 3$ haplotypes within the DRB1*1300 group could be rejected with a chisquare of $9.701(2 d f)$, corresponding to a $p$-value of 0.0078 . The situation is more complex however, because of the existing linkage disequilibrium of the DRB1*1300 alleles with different neutral or protective DQA1-DQB1 haplotypes (see below).

Analogous analysis of the DRB5 alleles (Table 3) showed a significant decrease, to the same extent as DRB1*1500, of DRB5*0101, among IDDM patients. This is a consequence of the strong linkage disequilibrium between the DRB5*0101 and DRB1*1501 alleles $[4,24]$.

Distribution of $D Q A 1-D Q B 1-D R B 1$ haplotypes. The distribution of the 33 deduced DQA1-DQB1-DRB1 haplotypes found in this study is shown in Table 5. Deduced haplotypes fitted with those expected from the reported linkage disequilibria $[4,24]$ for all except one control subject and one IDDM patient. A significant decrease of the DRB1*1500-DQA1*0102-DQB1* 0602, DRB1*1100-DQA1*0501-DQB1*0301, DRB1* 1300-DQA1*0103-DQB1*0603, DRB1*0701-DQA1* $0201-D Q B 1 * 0303$ haplotypes in the IDDM patient population was shown. The DRB1*0301-DQA1*0501DQB1*0201 and DRB1*0401-DQA1*0301-DQB1* 0302 haplotypes were however significantly increased among the diabetic patients. 
Table 3. Distribution of DRB1, DRB5 alleles among IDDM patients and healthy control subjects

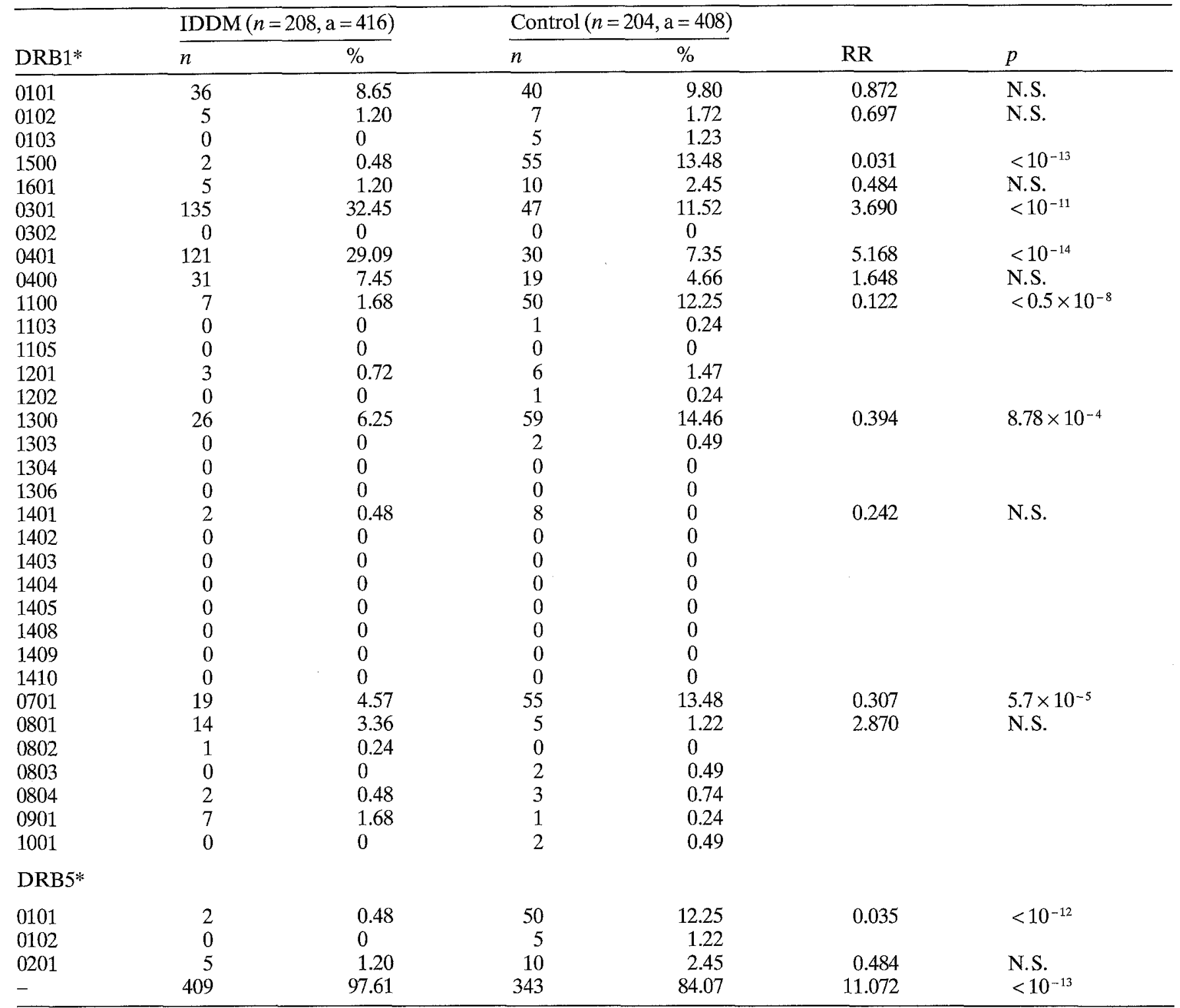

$P$-values were corrected for the number of DRB1 and DRB5 alleles tested. The DRB1*0400 group contains all DRB1*04 alleles except for DRB $1 * 0401$. The DRB $1 * 1100$ and DRB $1 * 1300$ groups contain the DRB1*1101/1102 and DRB1*1301/1305 alleles, respectively. All DRB alleles that were not counted at least 10 times in the total (control + IDDM) population were

All diabetic and control subjects who carry the DRB1*1500-DQA1*0102-DQB1*0602 haplotype encode the DRB5*0101 allele. In our assay we could not distinguish between the DRB $1 * 1501 / 1502 / 1503$ alleles. However, because of the reported linkage disequilibria for the DR2 alleles $[4,24]$, it is most likely that DRB $1 * 1501$ is present in this strong protective haplotype. Although DRB $1 * 1501 / 1503$ are both included in identical extended haplotypes, DRB $1 * 1503$ can probably be excluded because it is almost exclusively found in the black population $[4,31]$. It is also unlikely that the DRB1*1500 haplotype contains DRB1*1502 because not tested. Unresolved typing results (DRB1*0405/0408= DRB $1 * 0405 / 0405, \quad$ DRB $1 * 0401 / 0405=$ DRB $1 * 0408 / 0409$, DRB1*1201/DRB1*0101-DRB1*0102) were obtained for three IDDM patients and one control subject and were not included in the statistical analysis, a: number of diabetic and control chromosomes. N. S., not significant

this allele is probably included in the DRB5*0102 encoding DRB1*1500-DQA1*0103-DQB1*0602 and DRB1*1501-DQA1*0102-DQB1*0502 haplotypes, predominantly present in the oriental population [4,24].

By subdividing the DRB1*1300 group according to the extended DQA1-DQB1 haplotypes, the protective effect of DRB1*1300 seems to be mainly caused by the DQA1*0103-DQB1*0603 haplotype (RR for DRB1 $* 1300=0.394$ (Table 3), RR for DRB1*1300DQA1*0103-DQB1*0603 $=0.176$ (Table 5) . In accordance with the reported linkage disequilibrium [4], the DRB1*1300-DQA1*0103-DQB1*0603 haplotype 
was exclusively associated with the expression of the DRB3*0101 and DRB3*0200 alleles in our population. We concluded that the protective effect of the DRB1*1300-DQA1*0103-DQB1*0603 haplotype is strictly related to the expression of DRB $3^{*} 0101$ (data not shown). This is similar to what we observed for DRB1*0301, where susceptibility was related to the expression of $\mathrm{DRB}^{*} 0200$. These results for the DRB1 $* 1300$ haplotype allow us to suggest that DRB1*1301, rather than the DRB1*1302 or the DRB1*1305 alleles will be included in this protective haplotype. Although we can suggest the DRB1*1501 and DRB $1 * 1301$ alleles are responsible for the protective effect of the DRB $1 * 1501$ and DRB1*1300 groups of alleles, it should be mentioned that minor exceptions to the accepted linkage disequilibria have been reported $[4,24]$.

Interesting also is the DRB $1 * 07$ haplotype; whereas the DQA1*0201-DQB1*0201 haplotype clearly accounts for the previously reported neutral effect for the DRB $1 * 0701$ allele in the Caucasian population $[2,29]$, a protective effect was shown for the DR7 associated DQA $1 * 0201-D Q B 1 * 0303$ haplotype, indicating a protective role for the $\mathrm{Asp}^{57+} \mathrm{DQB} 1 * 0303$ allele.

Next we analysed the homogeneity of the RRs of the different DRB1*04 alleles and of the different DR4-associated DQB1 alleles among the DR4-positive patients and control subjects (Table 4). It should be mentioned that RRs differ from those presented in Table 2 (for the DQB1RR) and Table 3 (for the DRB1*04RR). Here, the RRs were calculated by comparison with the non-DR4 group (259 diabetic and 358 control chromosomes, respectively). Concerning the DQB1*03 group of alleles, an increased total risk of DQB $1 * 03$ was observed in the DR4 positive IDDM group (RR of 4.147). Homogeneity among the separate RRs for the different DQB $1 * 03$ alleles within the DR4-positive group could be rejected with a chi-square of $15.251(1 \mathrm{df})$, corresponding to a $p$-value of 0.0001 . Thus, we can conclude that the risk of DQB $1 * 0302$ was significantly increased among DR4 positive IDDM patients. Analogously, we tested the distribution of the DRB $1 * 04$ alleles among the DR4-positive group of patients and control subjects (Table 4). An increased total risk of DRB $1 * 04$ was observed in the DR4 positive IDDM group (RR of 4.147). Homogeneity among the separate RRs for the different DRB1*04 (DRB1*0401<-> DRB1*0400) alleles within the DR4 positive group could be rejected with a chi-square of $6.699(1 d f)$, corresponding to a $p$-value $<0.01$. Thus, we can conclude that the risk of DRB1*0401 was significantly increased among DR4 positive IDDM patients. In order to unravel the contribution of DRB1*0401 and DQB1*0302 [32], we applied a stepwise likelihood-ratio test. Evidence for effects of DQB1 (chi-square 13.398, $1 \mathrm{df}$ ) and DRB1 (chisquare $6.527,1 d f$ ) was strong. The assumption that both loci act as independent risk factors provided a substantially better explanation of the data (chi-square 6.527, 1
Table 4. Distribution of DRB $1 * 04$ alleles and DQB 1 alleles, DRB1*0301-DRB3 and DRB1*1300-DRB3 haplotypes among DRB1*04, DRB $1 * 03$ and DRB $1 * 13$ positive control subjects and IDDM patients, respectively

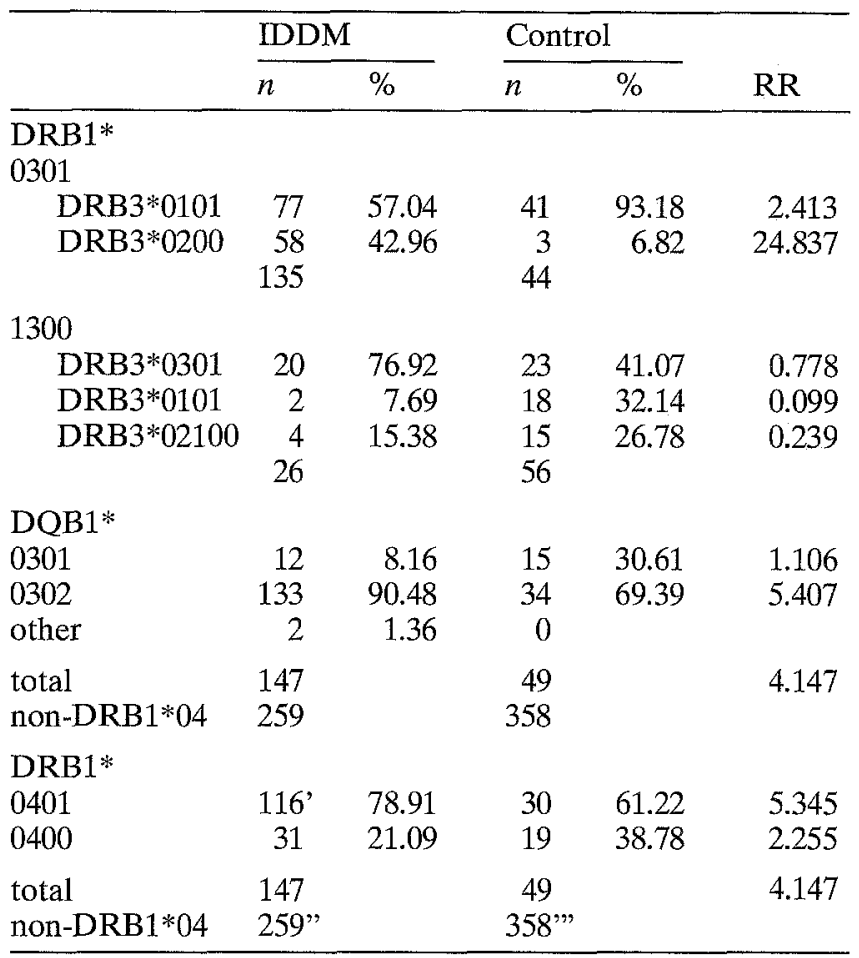

$P$-values were corrected for the number of alleles or haplotypes tested. The DRB1*0400 group contains all DRB1 *04 alleles except DRB1*0401. The DRB1*1300 group contains the DRB1*1301/1305 alleles. All alleles or haplotypes that were not counted at least 10 times in the total (control + IDDM) population were not tested. The DRB $1 * 0301-\mathrm{DRB} 3$ haplotype phase could not be assigned for three control persons that were not included in the analysis. The DRB $1 * 1300$-DRB3 haplotype phase could not be assigned for three control persons. ': of the 121 original IDDM DRB $1 * 0401$ alleles, five occurred in patients for whom no haplotype could be constructed. ": of the 264 original IDDM non-DRB1*04 DRB1 alleles, five occurred in patients for whom no haplotype could be constructed. ": of the 359 original non-DRB $1 * 04$ DRB1 alleles in the control population, one occurred in a control subject for whom no haplotype could be constructed. RRs were calculated by comparison with the total population - the respective group of alleles for which heterogeneity of the separate alleles or extended haplotypes was tested

$d f$ ) than the assumption that DRB1*0401 is not a risk factor but simply in linkage disequilibrium with DQB $1 * 0302$ (chi-square $0.110,1 d f$ ). The most complex hypothesis, in which both alleles act as risk factors, while a different disequilibrium between both alleles in the IDDM and control population exists provided only a marginally better explanation than the hypothesis in which both alleles act as independent risk factors that are not in disequilibrium (chi-square 1.473, $2 d f$ ). Thus, we can confirm the hypothesis of Sheehy et al. [32] that the DRB $1 * 0401$ and DQB $1 * 0302$ alleles confer a high risk of developing IDDM and that they act as independent risk factors. 
Table 5. Distribution of DQA1-DQB1-DRB1 haplotypes among IDDM patients and control subjects

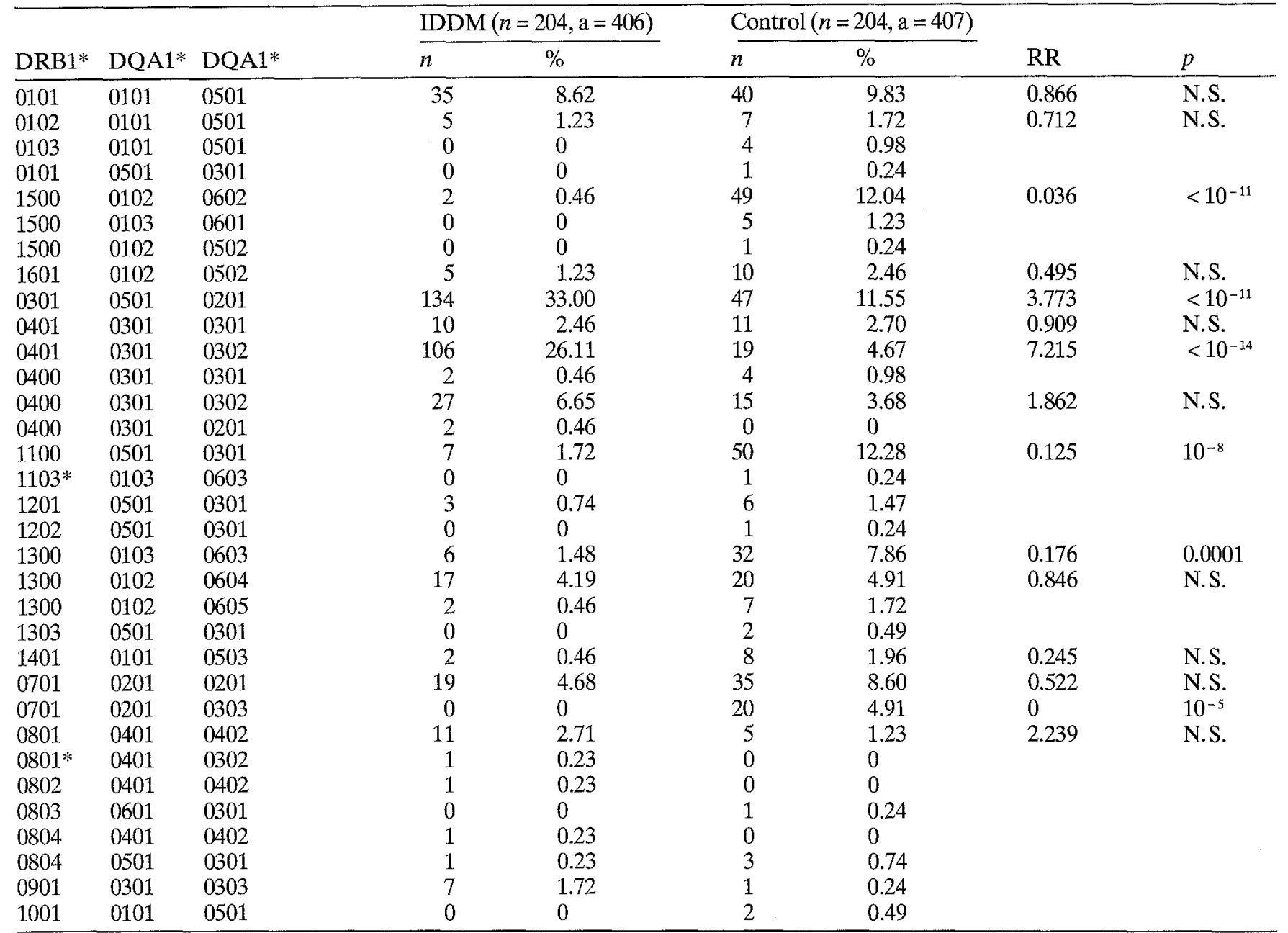

$P$-values were corrected for the number of haplotypes tested. The DRB $1 * 0400$ group contains all DRB $1 * 04$ alleles except for DRB1*0401. The DRB1*1100 and DRB1*1300 groups contain the DRB1*1101/1102 and DRB1*1301/1305 alleles, respectively.

Probable DRB1-DQA1-DQB1 haplotypes that have not yet been described are indicated by *. All DRB1-DQA1-DQB1 haplotypes that were not counted at least 10 times in the total (control + IDDM) population were not tested. Typing results were not included for seven IDDM patients (DQB1 and DRB typing

We also confirmed the previously suggested hypothesis that particular DQA1-DQB1 haplotypes, rather than specific DQA1 or DQB1 alleles, are associated with IDDM $[9,10]$. Analysis of the IDDM associated haplotypes showed that the effect of particular increased (susceptibility: $S=D Q A 1^{A r g 52+}$ or DQB1 ${ }^{\text {Asp57-) }}$ or decreased (protective: $\mathrm{P}=$ DQA1 ${ }^{\mathrm{Arg} 52-}$ or DQB1 ${ }^{\mathrm{Asp} 57+}$ ) DQA1 or DQB1 alleles was caused by their association with particular $\mathrm{S}$ or $\mathrm{P}$ DQB1 or DQA1 alleles, respectively; the effect of the susceptible DQA $1 * 0301$ and protective DQA1*0201 alleles appeared to be related to their presence in the DQA $1 * 0301-\mathrm{DQB} 1 * 0302$ and DQA $1 * 0201-D Q B 1 * 0303$ haplotypes, respectively. results were missing for four and three patients, respectively) and one control person (the DRB typing result was missing) (cfr supra). The alleles DQA1*0401/0601 could not be distinguished in one control person and two IDDM patients, encoding DQA $1 * 0201$ on the second chromosome. For these persons, only the DQA1*0201 associated haplotype was included in the statistical analysis. Therefore, a total of 406 diabetic and 407 normal chromosomes were compared. $n$ : number of IDDM patients and control subjects, a: number of diabetic and control chromosomes. N.S.: not significant

Analogously, the effect of the susceptible DQB $1 * 0201$ and protective $\mathrm{DQB} 1 * 0301$ alleles seemed to be related to their presence in the DQA $1 * 0501-\mathrm{DQB} 1 * 0201$ and DQA $1 * 0501-\mathrm{DQB} 1 * 0301$ haplotypes, respectively (Table 5). The DQA1*0501 was increased or decreased among the IDDM patient population, depending on the particular DQA1-DQB1 haplotype that was formed and therefore the effect based on its allele frequency was neutralized.

Distribution of DQA1-DQB1 genotypes. Concerning DQB1 genotypes, it was demonstrated previously that homozygosity for non- $\mathrm{Asp}^{57} \mathrm{DQ} \beta$ chains is positively associated with IDDM [6-8]. A total of $74.9 \%$ of our 
Table 6. Frequencies among IDDM patients and control subjects of the different HLA-DQ genotypes, according to the number of susceptible (SS) DQA1-DQB1 haplotypes that are encoded in cis and/or trans

\begin{tabular}{lllc}
\hline SS DQA1/DQB1 & IDDM & Control & RR \\
heterodimers & $n=207$ & $n=205$ & \\
& $n$ & $n$ & \\
\hline 0 & 12 & 102 & 1.0 \\
1 & 22 & 52 & 3.596 \\
2 & 77 & 42 & 15.583 \\
4 & 96 & 9 & 90.667 \\
\hline
\end{tabular}

RR were calculated by comparison with the 0 dose group. $n$ : number of IDDM patients and control subjects

IDDM patients, compared to $27.3 \%$ of our control subjects were $\mathrm{DQB}^{57-}$ homozygous. Since we found a strong predisposing effect for $\mathrm{Asp}^{57-}$ alleles $(\mathrm{RR}=6.339$, Table 2), we wanted to evaluate whether the effect in individuals who are homozygous for Asp ${ }^{57-}$ is simply a consequence of the presence of two predisposing alleles or whether there is an additional increase in risk. In a likelihood-ratio test, the hypothesis that the RR in homozygotes would be equal to the square of the RRs in heterozygotes ( 40.18 vs 6.339 ) was compared to the hypothesis that each group has his own RR (allowing for interaction in homozygotes). No significant evidence could be obtained for an interactive effect in homozygotes (chi-square 1.258,1 df). The presence of $\mathrm{Asp}^{57-}$ alleles seems to be a risk factor that acts in a codominant fashion. Because of the heterogeneity observed among the predisposing effects of different DQB $1^{57-}$ alleles, we suspected an analogous heterogeneity among the RRs of the different DQB1 ${ }^{\text {Asp57- }}$ (SS) genotypes. The analysis of the DQB1 genotypes is in agreement with our previous conclusion, based on the genetic heterogeneity of $\mathrm{Asp}^{57-} \mathrm{DQB} 1$ alleles, that other risk factors are likely to act in tandem with the predisposing DQB1 genotypes. Most patients encoding SS genotypes carried the DQB1*0303/0302, DQB $1 * 0302 / 0201$ or DQB1*0201/0201 genotypes. Indeed, the highest RRs were obtained for the DQB $1 * 0302 / 0302$ and DQB1*0201/0302 genotypes (data not shown).

Khalil and co-workers [10] originally suggested that IDDM is associated with the combined presence of particular susceptible (S) DQA1 $\left(\mathrm{Arg}^{52+}\right)$ and susceptible (S) DQB1 $\left(\mathrm{Asp}^{57}\right.$ ) alleles. In our study, we also detected predisposing effects for particular DQA1 and DQB1 alleles. It has been suggested by Khalil et al. [11] that these factors interact in a specific way. Frequencies of the different HLA-DQ genotypes, according to the number of susceptible (SS) DQA1-DQB1 haplotypes that are encoded in cis and/or trans, among IDDM patients and control subjects, are indicated in Table 6. The highest risk $(\mathrm{RR}=90.667)$ was observed for those patients, encoding a DQA1-DQB1 genotype that gives rise to the maximum of SS heterodimers [4]. RR were calculated by comparison with the 0 dose group of per- sons $(\mathrm{RR}=1)$. We compared the scenario where both risk factors act independently with the scenario proposed by Khalil et al. [11], where the number of possible allelic interactions is regarded as a "dosis". A straightforward comparison of likelihoods was not possible, as the two scenarios do not represent nested models. Use of Akaikes information criterion [33] enabled us to compare the two hypotheses. The "dosis" hypothesis yielded a slightly better explanation (likelihood 1518.440) than the hypothesis of no interaction between $\mathrm{Asp}^{57-}$ and $\mathrm{Arg}^{52+}$ (likelihood 1518.965), but the difference is so marginal that a firm conclusion is not warranted.

\section{Discussion}

Using a highly discriminatory DNA typing technique and large populations of IDDM patients and control subjects, we were able to obtain more refined results on the association of IDDM with particular HLA class II alleles, haplotypes and genotypes.

In a first phase we analysed allelic associations for each polymorphic class II locus. We found a significant positive association for $\mathrm{Arg}^{52+}$ DQA1 and $\mathrm{Asp}^{57-}$ DQB1 alleles, as reported previously $[6-8,10]$. However, we could clearly show heterogeneity among the alleles comprised in each of these groups, only specific alleles being significantly associated. For the DPB1 gene, we found a very significant increase of the rare DPB $1 * 0202$ allele among IDDM patients. Analysis of the extended haplotypes for these patients, however, revealed that this association most likely occurred due to linkage disequilibrium of this allele with the DR3 serologic specificity, which positively associates with IDDM. More interesting results were obtained by analysing extended haplotypes and genotypes. As shown in Table 5, significant association was found for particular DRB-DQA1-DQB1 haplotypes. Specifically for the DR4-related susceptibility haplotype, we showed that it was the combined presence of DRB1*0401-DQA1*0301-DQB1*0302 alleles that was responsible for an increased risk. Moreover, we showed that each of the DRB1*0401 and DQB1*0302 alleles confer an increased risk but also that these alleles act as independent risk factors. For the DR3 specificity, we demonstrated that this effect was restricted to the DRB3*0200 encoding haplotype. The highest predisposing effect was obtained for those DQ genotypes that encode four SS heterodimers (S referring to DQA1 $\mathrm{Arg}^{52+}$ and DQB1 Asp ${ }^{57-}$ susceptibility alleles). We could not however obtain conclusive evidence of an interactive effect between the susceptible DQA1 and DQB1 alleles, as reported by Khalil et al. [11].

These data suggest that it is the specific appearance of particular alleles encoded at the various HLA class II loci that confers susceptibility or protection to IDDM. For the DR4 associated susceptibility we dem- 
onstrated that both alleles act as independent risk factors. However, how these different loci are involved in the development of IDDM remains unclear, as the nature and the role of possible (auto)antibodies that are responsible for the initiation of the immunologic destruction of pancreatic beta cells remains unclear. Finally, because of the multifactorial nature of IDDM, in which multiple genes and environmental factors are involved, a $100 \%$ HLA-IDDM association could not be obtained in this study. Nevertheless, very significant associations for a restricted number of haplotypes and genotypes were obtained, which could be used in predisposition analyses, particularly in familial cases.

Acknowledgements. This work was supported by a grant 'Geconcerteerde Acties' from the Belgian Government and by the Interuniversity Network for Fundamental Research sponsored by the Belgian Government (1991-1996). Mahdi Zamani Ghabanbasani has a grant of the Ministry of Science \& Higher Education of Iran. Peter Marynen is a "Bevoegdverklaard Navorser" of the "Nationaal Fonds voor Wetenschappelijk Onderzoek", Belgium.

\section{References}

1. Olmos P, A'Hern R (1988) The significance of the concordance rate for type 1 (insulin dependent) diabetes in identical twins. Diabetologia 31: 747-750

2. Thomson G, Robinson WP, Kuhner MK et al. (1988) Genetic heterogeneity, modes of inheritance, and risk estimates for a joint study of Caucasians with insulin-dependent diabetes mellitus. Am J Hum Genet 43: 799-816

3. Kappes D, Strominger JL (1988) Human class II major histocompatibility complex genes and proteins. Ann Rev Biochem 57:991-1028

4. Fernandez-Vina MA, Gao X, Moraes ME et al. (1991) Alleles at four HLA class II loci determined by oligonucleotide hybridization and their associations in five ethnic groups. Immunogenetics 34: 299-312

5. Trowsdale J, Ragoussis J, Campbell RD (1991) Map of the human MHC. Immunol Today 12: 443-446

6. Todd JA, Bell JI, McDevitt HO (1987) HLA-DQ $\beta$ gene contributes to susceptibility and resistance to insulin-dependent diabetes mellitus. Nature 329: 599-604

7. Morel PA, Dorman JS, Todd JA, McDevitt HO, Trucco M (1988) Aspartic acid at position 57 of the HLA-DQ $\beta$ chain protects against type I diabetes: a family study. Proc Nat1 Acad Sci USA 85: 8111-8115

8. Ronningen KS, Iwe T, Halstensen TS, Spurkland A, Thorsby $\mathrm{E}$ (1989) The amino acid at position 7 of the HLA-DQ $\beta$ chain and susceptibility to develop insulin-dependent diabetes mellitus. Hum Immunol 26: 221-225

9. Todd JA, Mijovic C, Jenkins D, Bradwell AR, Barnett AH (1989) Identification of susceptibility loci for insulin-dependent diabetes mellitus by trans-racial gene mapping. Nature 338: $587-589$

10. Khalil I, d'Auriol L, Gobet M et al. (1990) A combination of HLA-DQ $\beta$ Asp57-negative and HLA-DQ $\alpha$ Arg52 confers susceptibility to insulin-dependent diabetes mellitus. J Clin Invest 85: 1315-1319

11. Khalil I, Deschamps I, Lepage V, Al-Daccak R, Degos L, Hors J (1992) Dose effect of cis- and trans-encoded HLA-
DQO $\beta$ heterodimers in IDDM susceptibility. Diabetes 41 : 378-384

12. Buyse I, Decorte R, Baens Met al. (1993) Rapid DNA typing of class II HLA antigens using the polymerase chain reaction and reverse dot blot hybridization. Tissue Antigens 41: 1-14

13. Meyer CG, Gallin M, Erttmann K et al. (1992) DQB1*WA1a new DQB1 allele identified in West Africa. Tissue Antigens 39: 147-149

14. Tautz C, Zwollo P, Marsh DG, Baur X (1992) Sequence of a novel HLA-DQB1 allele. Immunogenetics 35: 421-424

15. Gao X, Veale A, Serjeantson S (1992) AB1: a novel HLADPB1 allele found in one third of an Australian population. Immunogenetics 36: 64-66

16. Bodmer JG, Marsh SGE, Albert ED et al. (1992) Nomenclature for factors of the HLA system, 1991. Tissue Antigens 39: $161-174$

17. Apple RJ, Erlich HA (1992) Two new HLA DRB1 alleles found in African Americans: implications for balancing selection at positions 57 and 86. Tissue Antigens 40:69-74

18. Gao X, Veale A, Serjeantson S (1992) HLA class II diversity in Australian Aborigines: unusual HLA-DRB1 alleles. Immunogenetics 36: 333-337

19. Scharf SJ, Griffith RL, Erlich HA (1991) Rapid typing of DNA sequence polymorphism at the HLA-DRB1 locus using the polymerase chain reaction and nonradioactive oligonucleotide probes. Hum Immunol 30: 190-201

20. Woolf B (1955) On estimating the relation between blood group and disease. Ann Hum Genet 19: 251-253

21. Fisher RA (1960) The design of experiments. Oliver \& Boyd, Edinburgh, p 258

22. Dunn OJ (1958) Estimation of the means of dependent variables. Ann Math Stat 29: 1095-1111

23. Dunn OJ (1961) Multiple comparisons among means. Am J Stat Assoc 56: 52-64

24. Gao X, Serjeantson SW (1991) Heterogeneity in HLA-DR2related DR, DQ haplotypes in eight populations in AsiaOceania. Immunogenetics 34: 401-408

25. Oudshoorn M, du Toit ED, Martell RW (1990) New HLADR2-related specificities detected in South African blacks and individuals of mixed ancestry. Tissue Antigens 35: 211216

26. Schreuder GMTh, van den Berg-Loonen PM, Verduyn W et al. (1991) Increasing complexity of HLA-D2 as detected by serology and oligonucleotide typing. Hum Immunol 32: 141-149

27. Bidwell JL, Bidwell EA, Dupont E et al. (1992) Molecular characterization of a recombinant HLA-DR1/DR2 haplotype. Hum Immunol 33: 289-293

28. Buyse I, Emonds M-P, Bouillon R, Marynen P, Cassiman J-J (1993) Novel class II HLA-DRB4 and DPB1 alleles found in the Belgian population. Immunogenetics $38: 380$

29. Mijovic CH, Barnett AH, Todd JA (1991) Trans-racial gene mapping studies. Baillières Clin Endocrinol Metabol 321340

30. Ronningen KS, Spurkland A, Iwe T, Vartdal F, Thorsby E (1991) Distribution of HLA-DRB1, -DQA1 and -DQB1 alleles and DQA1-DQB1 genotypes among Norwegian patients with insulin-dependent diabetes mellitus. Tissue Antigens 37: 105-111

31. Demopulos JT, Hodge TW, Wooten V, Acton RT (1991) A novel DRB1 allele in D2-positive American Blacks. Hum Immunol 30: 41-44

32. Sheehy MJ, Scharf SJ, Rowe JR et al. (1989) A diabetes-susceptible HLA haplotype is best defined by a combination of HLA-DR and -DQ alleles. J Clin Invest 83: 830-835

33. Akaike H (1974) A new look at the statistical model identification. IEEE Trans 19: 716-723 